\title{
Glucose intolerance, diabetes mellitus and atherosclerosis; prospects for prevention
}

\author{
HaRRY KeEN \\ M.D., F.R.C.P. \\ Unit for Metabolic Medicine, Diabetic Clinic, Guy's Hospital, London, S.E.1
}

\begin{abstract}
Summary
Increased prevalence and incidence of arterial disease has become the major cause of disability and death in diabetics in Western cultures-but is much less common among Japanese and African diabetics. In the West, the presence of lesser, 'subdiabetic' degrees of glucose intolerance are probably independent 'risk factors' for accelerated atherosclerosis. Although many explanations for the link have been advancedamong them direct effects of glucose and insulin on the arterial wall, less direct effects of circulating lipoproteins, abnormalities of blood clotting and fibrinolysis and elevated blood pressure, even the influence of antidiabetic treatment itself-none has been clearly established. Nevertheless there is scope and hope that appropriate measures may reduce the raised arterial disease risk in the diabetic.
\end{abstract}

ThOSE of us who are involved in the clinical treatment of diabetes know well that vascular disease is the major unsolved problem which now confronts us in its management. Maintaining active life with insulin in youthful-onset patients, and the relief of symptoms and lowering of blood sugar levels with diet and oral antidiabetic agents in maturity-onset cases is not too difficult. But despite these measures of metabolic control our diabetic patients continue to develop vascular disease, affecting vessels from capillary to arterial calibre. In western societies, for instance, diabetic retinopathy is the single major registrable cause of blindness in middle-aged people. From the diabetic population comes a disproportionately high number of people admitted to hospital after myocardial infarction; and a fatal outcome is more likely in them than in non-diabetics. Diabetics are also greatly over-represented among those suffering from angina pectoris, intermittent claudication, gangrene and stroke. This paper will deal generally with 'arterial disease' rather than exclusively with coronary artery disease, for although ischaemic disease of the lower limbs is probably proportionately more common in the diabetic it is very likely that the statements made below are applicable to the heart as well.
These changing problems of the diabetic are reflected in the mortality statistics of the Joslin Clinic which span the era 1900-1970, and which classify proportions of death by major causes in the diabetics under their care over this time period (Marks, 1971). In the pre-insulin era diabetic coma was the preeminent cause of death. The advent of insulin of course greatly reduced its importance but it has been more than supplanted by the curious composite category, cardio-renal vascular disease, which has increased till it now accounts for about $80 \%$ of the total deaths of diabetics. However, this portrayal is not exactly what it seems. The types of patient regarded as diabetic in the pre-insulin era were different from the patients seen in greatest number in diabetic clinics today. Now a very high proportion of clinic attenders are the older, so-called maturityonset type of diabetic to whom Osler (1912) was loath to apply the term 'diabetic' at all, preferring to call them 'glycosurics'.

The relationship between arterial disease and diabetes mellitus can be viewed in different ways. Estimates may simply be made of manifest arterial disease in patients with clinically evident diabetes; or the question can be more widely interpreted, extending the study of the relationship further into the wider group of glucose intolerant subjects (which will obviously include the known diabetics) and seeking additional, less obvious (and sometimes less convincing) manifestations of arterial disease (e.g. questionnaire symptoms, ECG changes). Further information may be gained from a consideration of disturbance of glucose metabolism in subjects with arterial disease. Having established the nature of the relationship, one may consider mechanisms to which it might be attributable; and these can conveniently be divided into those which closely relate to glucose intolerance and those which are not so obviously related.

\section{Arterial disease in clinical diabetics}

Perhaps even now, the view that diabetics are particularly susceptible to arterial disease of heart, leg and brain is not without its challengers. Pyke 
(1968) based his scepticism on the ease with which false assumptions about relationships between diseases can be made in the absence of adequate comparative control evidence. Mitchell and Schwartz (1965) were also unable to find convincing evidence of a special association between diabetes and atherosclerosis in their classical post-mortem study. However, the accumulation of data from a wide variety of other sources makes it difficult to refute the special relationship.

The International Atherosclerosis Project (Robertson and Strong, 1968), was a standardized, semiquantitative study of atherosclerosis found in over 23,000 post-mortem studies in eighteen defined population groups. It concerned itself particularly with the amount of fatty streaking and raised atherosclerotic lesions in the arterial wall. In comparisons of findings in diabetics versus non-diabetics, in the coronary arteries of men aged 45-54, there was a clear excess of both types of lesion in diabetics in all of the population groups compared. It is noteworthy, however, that in countries with a generally low prevalence of arterial disease (e.g. Costa Rica and Mexico) the prevalence in diabetics was also low, though higher than in local non-diabetics. In a well constructed long-term study of morbidity and mortality among a cohort of $\mathbf{3 5 0}$ diabetics working for the Dupont Company in the United States, compared with an equal number of non-diabetic employees matched for age, sex and job status, Pell and d'Alonzo (1970) found that over a ten-year period, forty-five diabetics died of coronary heart disease compared with sixteen controls. In addition to this statistically significant three-fold excess of CHD deaths there was a similar excess of cerebrovascular deaths although, in this category, numbers were small and the difference did not achieve significance. Similar findings emerge from the classic Framingham study (Garcia et al., 1974) where a cohort of town dwellers have been followed over the course of now 16 years. Comparisons have been made of both cardiovascular morbidity and mortality in diabetics aged 30-62 years at entry to the study. Diabetics show a considerable increase in incidence of coronary heart disease, stroke and claudication compared with rates for the population as a whole. For coronary heart disease, this probably is an underestimate for diabetics, because up to a third of attacks of myocardial infarcation may be painless and so escape recognition. The study of the outcome of myocardial infarcation in diabetics (Soler et al., 1975) reported from Birmingham, recorded a mortality rate of $39.7 \%$ for those admitted to hospital, a fatal coutcome about twice as likely as for a non-diabetic, although somewhat less unhappy conclusions have been arrived at by others. In Framingham, a two-and-a-half-fold enhanced mortality from coronary disease was recorded, an excess that was most marked in the insulin-treated patients. It is $\underset{Z}{Z}$ noteworthy that coronary mortality rates were $\stackrel{\mathbb{\complement}}{\varrho}$ rather higher in the diet-treated patients than in $c$ those treated with oral antidiabetic agents, a finding $\vec{F}$ which gives no support to the dangers supposedly associated with oral agents (University Group? Diabetes Program, 1970 and 1975, see below).

What is true for diabetes and arterial disease in the $\frac{\bar{c}}{7}$ U.S.A. appears not to hold for certain other areas of $\mathbb{\Phi}$ the world. A collection of somewhat fragmentary $\infty$ data published in Japan suggested that there, $\vec{O}$ coronary mortality rates were much lower for nondiabetics and diabetics alike and this has been $\vec{\omega}$ supplemented by the publication of a large collection $\frac{\rho}{\circ}$ of data from 3151 post-mortems in diabetics by Goto, Sato and Masuda (1974). Two interesting and 3 . important points emerge from their figures, first, the N low frequency of death attributed to coronary heart 9 disease in Japanese diabetics $(6.5 \%)$, compared with $₫$ $53.1 \%$ of American diabetic deaths in 1960-66 (Bradley, 1971); second, although rates of 'coronary $\mathrm{G}$ death' are also low among the Japanese general population, rates in diabetics are about twice as high $\vec{c}$ as for non-diabetics. This is so too for cerebral infarction as a cause of death but not, interestingly enough, for cerebral haemorrhage, which runs of about the same rate for diabetics as for the gener population. Age at death and duration of diabet differ a little between the U.S.A. and Japanese diabetic post-mortem groups but cannot account for more than a fraction of the CHD difference and will certainly not affect the general conclusion that $\stackrel{\mathbb{Q}}{\stackrel{2}{ }}$ arterial disease is a strikingly less important cause of $\overrightarrow{\overrightarrow{0}}$ death in Japanese than in Western diabetics. On the $\frac{9}{3}$ basis of this and the other prima facie evidence of large differences between the behaviour of diabetic groups in various parts of the world in respect of cardiovascular morbidity and mortality, a standar- 3 . dized comparative study of vascular morbidity in $\dot{\sigma}$ national groups of diabetics in many countries 3 . (including the U.K. and Japan) has been inaugurated $\delta$ under the auspices of the World Health Organization.

\section{Glucose tolerance and 'atherosclerosis indicators'}

Population studies of diabetes and glucose intolerance over the last two decades commend a rather different approach to the question of the blood $\sigma$ glucose/arterial disease link. When glucose tolerance $\tilde{O}$ is measured in large sections of the general popula- స్ట tion, wide variation in blood glucose response is found, varying between clearly diabetic responses at 0 the upper extreme and the great majority with $\stackrel{\mathbb{D}}{\mathbb{C}}$ normal responses at the other. Although there is a $\stackrel{\infty}{+}$ progressive loss of glucose tolerance with advancing 70 years, at all ages there appears to be a unimodal distribution of blood glucose responses and the 
diagnosis of diabetes mellitus is made at a 'cutting point' arbitrarily imposed on these distributions (Keen and Jarrett, 1976). In other words, rather than defining diabetes as a quality, one may regard glucose tolerance as a quantity, distributed in populations much like body weight or blood pressure, with diabetes representing an arbitrarily defined, well elevated, upper segment of the distribution, comparable to 'hypertension' and 'obesity' at the upper reaches of the arterial pressure and weight distribution respectively. In these terms, after systematic glucose tolerance measurements, about $85 \%$ of a Western population would be designated as clearly normal, $2-3 \%$, perhaps $5 \%$ (depending on its age and sex composition) as clearly diabetic, with 10-15\% falling into a borderline group, the size of which will depend upon the criteria defining its upper and lower limits. Following a large scale population survey in Bedford, estimates were made of the numbers with arterial disease in representative samples of normoglycaemic, newly-detected borderline diabetic and diabetic individuals (Keen et al., 1965). These estimates were based upon application of the standard WHO questionnaire for angina pectoris, history of myocardial infarction, claudication, frequency of completed stroke and Minnesotacodable ECG abnormalities. The frequency of arterial disease in these groups (i.e. the proportion of individuals with positive questionnaire responses or with ECG findings) increased stepwise with increasing degrees of glucose intolerance. It was about twice as high in diabetics as in normals with rates for borderline individuals lying between the two. Since the metabolic characterization was made at about the same time as the arterial disease assessment, the latter cannot be attributed to a knowledge of or treatment received for diabetes.

In the same three glycaemia groups, the incidence of new clinical arterial events was reassessed in 1967, i.e. over a 5-year period (Keen, 1971). This estimate of incidence included deaths certified as cardiovascular in cause. A clear and considerable gradient of increasing risk with increasing baseline glucose intolerance was shown. Even within the 'borderline diabetic' group there was a highly significant, twofold gradient of arterial disease incidence between those lying below and those lying above the median $2 \mathrm{hr}$, post-glucose sugar level. A shallower gradient relating arterial disease risk to random blood sugar measurements was found in the Framingham followup. Keen, Fuller and Jarrett (1976) have recently derived further data from a mortality study of a large group of male civil servants screened for glucose tolerance with a single $2 \mathrm{hr}$ post-load sugar and followed for 5 years. In this group, a clear predictive effect of blood glucose for increased cardiovascular mortality appeared to be independent of age, blood pressure, body weight, blood cholesterol and smoking history. Similar indications that glucose intolerance is a 'risk-factor', predictive of present or future arterial disease, have been found by Epstein (1973) who reviewed the findings of the studies in Tecumseh and elsewhere.

\section{Mechanisms of the association}

There is no lack of plausible explanation for the link between glucose metabolism and arterial disease although none has been clearly established. Hyperglycaemia may be simply and directly involved in arterial damage by way of activation of the sorbitol pathway (Morrison, Clements and Winegrad, 1970). This pathway has also been invoked to explain cataract and peripheral neuropathy in diabetics but, although the necessary enzymatic apparatus has been demonstrated in aortic endothelium, its role in human atherogenesis remains hypothetical. It has been suggested that localized accumulation of the poorly diffusible glucose alcohol, sorbitol, may cause endothelial swelling. Focal anoxia due to this local barrier to oxygen diffusion may affect permeability and perhaps allow increased local insudation of plasma lipoproteins, so inaugurating the atherosclerotic lesion.

Also produced in excess in the aorta of the human diabetic, glycosaminoglycans may cause local entrapment of lipoprotein by physico-chemical forces (Gerö, Bihari-Varga and Virag, 1973) which has been picturesquely likened to the entanglement of barbed wire strands in a briar hedge.

Elevation of blood glucose or loss of glucose tolerance is itself related to a number of other endocrine and metabolic factors which may be implicated in atherogenesis. The nature of the link with circulating insulin levels is a good example of this. With increasing degrees of glucose intolerance, circulating insulin levels rise, pari passu, although increasingly inadequately. Beyond a certain point, the failing insulin response breaks down completely and subnormal insulin levels occur. Both of these response phases may favour atherogenesis; hyperinsulinaemia will increase the production of triglyceride-rich lipoprotein (Reaven et al., 1967), will inhibit tissue lipolysis and stimulate in situ lipogenesis (Stout, 1971). Insulin may also have a direct stimulatory effect (Stout, Bierman and Ross, 1975) on the proliferation and perhaps other metabolic activity of the smooth muscle cells of the arterial media which may invade the intima and contribute to the focal lipid accumulations which culminate in the atheromatous plaque (Ross and Glomset, 1973). Hypoinsulinaemia is associated with depleted levels of adipose tissue lipoprotein lipase activity (Bierman, 1974) and this will slow the removal of lipoproteins from the circulation. Unfettered tissue lipolysis will 
increase circulating fatty acid levels and thereby enhance hepatic triglyceride synthesis. The preliminary results in thirty-five randomly selected insulin-dependent diabetics also suggest that activity of the cholesterol esterifying enzyme, lecithin cholesterol acyl transferase (LCAT) is significantly reduced in diabetics compared to normals, perhaps altering the properties of circulating lipoproteins and the mobilization and transport of cholesterol (Mattock et al., 1976).

Evidence for clinical associations between increased circulating insulin levels and atherosclerosis in man comes from the metabolic studies of Tzagournis and colleagues in individuals with premature coronary artery disease (Tzagournis et al., 1968). A high proportion of these non-diabetic individuals showed abnormally elevated plasma insulin responses to a glucose stimulus compared with very much lower levels among the normal controls. Other workers have produced supporting evidence for raised circulating insulin levels in otherwise apparently metabolically normal people with arterial disease, a relationship reviewed by Stout and Vallance-Owen in 1969. One must consider, however, the possibility that hyperinsulinaemia is a consequence rather than a cause of atherosclerosis. Glucose utilization, hindered by the mechanical obstruction of the arterial disease, will perhaps only return to normal at the expense of increased insulin production and action at unobstructed sites, a mechanical model of peripheral insulin resistance.

Despite a compendious literature, a systematic abnormality of circulating lipoproteins has not been clearly established as an integral feature of diabetes mellitus. Such abnormalities as do occur may be related to accompanying obesity, to the degree of metabolic uncontrol, to the nature of the therapeutic diet prescribed and perhaps to the drugs used in treatment. The characteristic abnormality of plasma lipids in diabetics, when it does occur, is the elevation of triglyceride-rich lipoproteins and it has been suggested that there may be qualitative (Schonfeld et al., 1974) as well as quantitative changes in the composition of circulating lipoproteins in the diabetic. The atherogenic potential of circulating lipoproteins in general and particularly the independent effect of the triglyceride-rich lipoproteins remains controversial and has been extensively considered by other contributors to this symposium. Their role in accentuating the arterial disease of the diabetic has been strongly advocated by some workers and it seems likely to be at least a contributory factor. The strength of the association between degrees of glucose tolerance/intolerance and fasting plasma triglyceride levels was well shown in a study of 220 subjects stratified by age, sex and adiposity from a normal working population (Abrams et al., 1969). The high degree of positive correlation between the fasting triglyceride concentration and the 'area' under the oral glucose tolerance curve ( $r$ between 0.7 and 0.8 ) suggested a possible mechanism for the link between glucose metabolism and arterial disease in the population at large. Perhaps, cautiously, it may be extrapolated into the higher, more clearly pathological ranges, of glucose intolerance.

Another facet of the relationship between diabetes and hyperlipidaemia is the well recognized frequency of impaired glucose tolerance, sometimes amounting to treatable diabetes, in several of the hyperlipoproteinaemias, especially those characterized by hypertriglyceridaemia of endogenous origin. The explanation that circulating triglyceride antagonizes the peripheral effects of insulin has been advanced by some (Davidson and Albrink, 1965) but challenged by the experimental work of others (Gibson et al., 1974). The common factor which may account for much of the association between hyperlipidaemia, hyperinsulinaemia and hyperglycaemia, is obesity. Unquestionably, weight reduction by diet will promptly correct these and other metabolic aberrations on many (though not all) occasions. This 'metabolic normalization' is often advanced as the justification for recommending therapeutic weight loss in the prevention of atherosclerosis, although its effectiveness in this respect has not been establishee in appropriate clinical trials.

A number of other factors potentially contributor to the heavy toll of atherosclerotic disease are to be found associated with diabetes. An unexplained anomaly is that there is a clear association between raised blood pressure and glucose intolerance found in population surveys but that, with a few exceptions, there is no systematic elevation of blood pressure in populations of established diabetics when compared with appropriate age and sex-matched normal controls (Keen, Track and Sowry, 1975). The hypertension of survey-discovered diabetics (Table 1) cannot be ascribed to age or obesity. It may denote the liability of certain individuals to over-react to

TABle 1. Mean blood pressure $(\mathrm{mmHg})$ in 'survey' and 'known' diabetics

\begin{tabular}{cccc}
\hline & \multicolumn{3}{c}{ Age (years) } \\
& $40-49$ & $50-59$ & $60+$ \\
\hline SP & $132 / 83$ & $138 / 85$ & $146 / 87$ \\
BD & $142 / 88$ & $147 / 90$ & $156 / 92$ \\
ND & $142 / 93$ & $152 / 94$ & $155 / 92$ \\
KD & $133 / 81$ & $139 / 82$ & $144 / 84$ \\
\hline
\end{tabular}

Mean systolic/diastolic pressures from a survey population (SP) of 19,161 males aged 40 or more of whom 194 were newly found 'borderline' diabetics (BD) with $2 \mathrm{hr}$ post glucose blood sugar 110-200; 49 were newly found diabetics (ND) with $2 \mathrm{hr}$ blood sugar $>200$; and 181 were known diabetics (KD). 
TABLE 2. Haemostatic variables in controls and diabetics (mean values \pm s.d.)

\begin{tabular}{|c|c|c|c|c|}
\hline & \multicolumn{2}{|c|}{ Male } & \multicolumn{2}{|c|}{ Female } \\
\hline & Control (745) & Diabetic (27) & Control (310) & Diabetic (30) \\
\hline Fibrinogen $(\mathrm{mg} / 100 \mathrm{ml})$ & $264 \pm 53$ & $291 \pm 52$ & $277 \pm 57$ & $298 \pm 50$ \\
\hline Factor VIII $(\%)$ & $99 \pm 35$ & $136 \pm 86$ & $97 \pm 38$ & $140 \pm 54$ \\
\hline $\begin{array}{l}\text { Fibrinolytic activity } \\
\text { (100/DBCLT) }\end{array}$ & $30 \pm 15$ & $24 \pm 13$ & $30 \pm 15$ & $20 \pm 12$ \\
\hline Platelet adhesiveness $(\%)$ & $41 \pm 17$ & $47 \pm 17$ & $44 \pm 18$ & $43 \pm 16$ \\
\hline
\end{tabular}

environmental stimuli of various sorts with supranormal, sustained blood pressure and blood glucose elevation. In this, the role of neuro-humoral autonomic responses and the pattern of endocrine, metabolic and vascular changes they induce must surely be relevant. The degree of blood pressure elevation involved is not trivial and could contribute 'en masse' to a distinct increase in arterial disease risk.

The question of abnormal blood clotting, clot lysis and platelet behaviour in diabetics has also been questioned through the years in relation to enhanced atherosclerotic disease. Fearnley and Chakrabarti have reported increased fibrinolytic activity in diabetic blood (Fearnley, Chakrabarti and Avis, 1963; Chakrabarti and Fearnley, 1974) and more recently, O'Malley et al. (1975) have produced strong evidence of increased platelet aggregation in diabetics with neuropathy. Together with King's College Hospital Diabetic Clinic, the Unit for Metabolic Medicine at Guy's is at present involved in a multinational comparative study of vascular disease in a representative sample of British diabetics; in collaboration with Dr T. Meade, Dr R. Chakrabarti and the staff of the Epidemiology and Medical Care Unit, Northwick Park, we are making systematic measurements in this group of coagulation, fibrinolytic and platelet factors. The increased fibrinogen and factor VIII concentrations and the markedly diminished fibrinolysis in the diabetics compared with the large control group of normal factory workers is shown in Table 2. These data are only preliminary and we cannot yet say whether the phenomenon is general or restricted to a subclass of the diabetics; but the large and highly significant mean differences support the view that increased thrombogenesis and diminished thrombolysis may play some role in the heightened risk of arterial disease in diabetics.

Finally, the uncomfortable possibility that treatment itself may play a part in atherogenesis must be considered. In recent years, the oral antidiabetic agents have come under suspicion. There is no suggestion of increased vascular disease with these preparations; in fact some studies suggest that they may be beneficial in this respect (Keen, Jarrett and Fuller, 1974; Keen et al., 1975). The current uncertainties, however, arise from the widely publicized findings of the University Group Diabetes Program $(1970,1975)$ suggesting that cardiovascular death occurred two to three times more frequently in maturity-onset diabetics treated with the oral antidiabetic agents, tolbutamide and phenformin than in a group treated with placebo. The findings of this monumental multicentre trial have been vigorously contested over the last few years (Acta diabetologica latina, 1974) on the grounds of flaws in experimental design, non-comparability of diabetics assigned to the various treatment groups and the anomalous and inexplicable absence of coronary disease attributed as a cause for the diabetics who died in the placebo group. The failure of several other random-allocation, double-blind trials of tolbutamide and phenformin to give even a hint of support to the UGDP mortality findings and the absence of suggestive trends in groups of 'free-living' diabetics carefully observed over long time periods (see for example the Framingham experience Garcia et al., 1974) only adds to the uncertainty surrounding this important question, one which may never be fully resolved. In any case, data on cardiovascular morbidity (as distinct from mortality) show no adverse effects of oral agents and, in some cases, provide suggestive evidence of benefit.

What is perhaps more open to question is the influence of the therapeutic diet prescribed for diabetics. In most westernized countries, the proportion of carbohydrate in the diabetic regime is of the order of $30-40 \%$ of total food energy; $15-20 \%$ comes from protein, and the rest, $40-55 \%$, is from fat. Customarily, the diabetic has been advised to 'fill up' on dairy products, butter, cream, cheese, eggs and lard which some might regard as an 'atherogenic' diet. These suspicions receive indirect support from 
the observation that arterial disease is strikingly less common among Japanese diabetics in Japan where $60 \%$ or more of daily food energy is taken as carbohydrate, the smaller fat component containing a large element of vegetable and marine oils. The dietary prescription cannot be the whole story, because evidence of cardiovascular morbidity is commoner in diabetics than in the general population actually at the time of diagnosis. This may point to an enhanced susceptibility of the diabetic's metabolic pattern to atherogenic dietary (or other) factors which are afflicting the population as a whole, a situation analogous to the diabetic's special susceptibility to tuberculosis when the agent of the disease was widespread a generation ago. The control of tuberculous infection generally has freed the diabetic from this excess hazard. One may reasonably hope that the identification and control of atherogenic factors will confer this additional benefit on the diabetic too.

\section{Conclusion and recommendations}

It is fitting to conclude on an optimistic and positive note. Atherosclerosis need not be thought an inherent part of the diabetic syndrome, in the same way, perhaps, as is microvascular disease. Stimulated by the example of the Japanese and the African diabetic, we should surrender our fatalistic views on arterial disease in diabetes and seek to eradicate this affliction which accounts for much of the illness and most of the deaths in diabetics. Perhaps the most important argument for the earliest diagnosis of diabetes is that it will provide the earliest opportunity for the application of anti-arterial disease measures in people whom we should regard as unduly susceptible. What should we do? There can be little argument about the potential value of weight reduction, correction of hypertension even of lesser degree, encouraging physical activity, stopping smoking and lowering of plasma lipids. It would seem reasonable to change the composition of the diet, increasing the proportion of carbohydrate to $50-60 \%$ at the expense of fat intake, substituting a good proportion of the latter with oils and fats high in polyunsaturated fatty acid content, advice that some would like to see applied to the whole population. Hyperglycaemia itself should not be forgotten although in most maturity-onset diabetics it is likely that the diabetes will disappear if the earlier recommendations are met. Should it persist, the intelligent use of oral agents or, in the rare patient, insulin, will control that problem too. To the traditional urine tests and blood glucose follow-up should be added systematic monitoring of other arterial disease risk factors-plasma lipids and blood pressure for example - with vigorous action taken to correct them, with drugs if necessary.
In many ways, the discovery of diabetes may be regarded as a signal to activate the physician and to motivate the patient to accept the changes in life style and the treatment aimed at preventing arterial disease. However, there is no conclusive demonstration that any or all of the measures recommended above will be effective in achieving this goal. The division of professional opinion on the question of the diabetic diet is still such as to make it ethically possible to start clinical trials to compare the 'low fat diet' with conventional dietary advice. To demonstrate a halving in cardiovascular death rates in five years with only a $1 \%$ likelihood that the difference is due to chance will require about 1000 diabetics in each diet group. The nucleus of a multicentre enterprise of this sort has already been established by Dr Hockaday, Dr Mann and Dr Turner in Oxford.

Among the persons most vulnerable to arterial disease in Western society is the middle-aged diabetic woman. She is six or seven times more likely to suffer from claudication, angina, cerebrovascular disease or myocardial infarction than her non-diabetic sister. It is she who would benefit most dramatically from the successful control of atherosclerosis. We could expect only to be congratulated in depriving her of equality with the male in this respect.

\section{References}

Abrams, M.E., Jarrett, R.J., Keen, H., Boyns, D.R. \& Crossley, J.R. (1969) Oral glucose tolerance and related factors in a normal population sample. II. Interrelationship of glycerides, cholesterol and other factors with the glucose and insulin response. British Medical Journal, 1, 599.

Acta diabetologica latina (1974) Misunderstanding or error? The UGDP report after four years. Ed. S. Marigo. Co-eds L. Adezah, P. Brunetti, G. Pozza, L. Robba and G-C. Urbinati. 11 (5), 357.

Bierman, E.L. (1974) Insulin levels and plasma triglyceride removal in diabetes. In: Diabetes. Proceedings of the Eighth Congress of the International Diabetes Federation, p. 360. Excerpta Medica, Amsterdam.

BRADLEY, R.F. (1971) Cardiovascular disease. In: Joslin's Diabetes Mellitus (11th Edition) (Ed. by A. Marble, P. White, R. F. Bradley \& L. P. Krall), p. 446. Lea \& Febiger, Philadelphia.

Chakrabarti, R. \& Fearnley, G.R. (1974) Pharmacological fibrinolysis in diabetes mellitus. Diabetologia, 10, 19.

Davidson, P.C. \& AlBRink, M.J. (1965) Insulin resistance in hyperglyceridemia. Metabolism, 14, 1059.

EPSTEIN, F.H. (1973) Glucose intolerance and cardiovascular disease. Triangle, 12, 3.

Fearnley, G.R., Chakrabarti, R. \& Avis, P.R.D. (1963) Blood fibrinolytic activity in diabetes mellitus and its bearing on ischaemic heart disease in obesity. British Medical Journal, 1, 921.

Garcia, M.J., McNamara, P.M., Gordon, T. \& Kannel, W.B. (1974) Morbidity and mortality in diabetics in the Framingham population. Sixteen year follow-up study. Diabetes, 23, 105.

\section{志}


然 
Gerö, S., Bihari-Varga, M. \& Virag, S. (973) Studies in the functional properties of vascular mucopolysaccharides. In: Vascular and Neurological Changes in Early Diabetes (Ed. by R. A. Camerini-Davalos \& H. S. Cole), p. 103. Academic Press, New York.

Gibson, T., Fuller, J.H., Grainger, S.L., Jarrett, R.J. \& KEEN, H. (1974) Intralipid triglyceride and oral glucose tolerance. Diabetologia, 10, 97.

Goto, Y., SAto, S-I. \& Masuda, M. (1974) Causes of death in 3151 diabetic autopsy cases. Tohoku Journal of Experimental Medicine, 112, 339.

Keen, H., Rose, G.A., Pyke, D.A., Boyns, D.R., ChlouVERAKIS, C. \& MISTRY, S. (1965) Blood sugar and arterial disease. Lancet, ii, 505.

KeEN, H. (1971) Factors influencing the progress of atherosclerosis in the diabetic. Acta diabetologica latina, 8 (suppl. 1), 444.

KeEN, H. \& JARRETT, R.J. (1976) Epidemiology of diabetes. In: Diabetes Mellitus: Diagnosis and Treatment. Vol. 4 (Ed. by K. E. Sussman \& R. J. S. Metz). American Diabetes Association Inc., New York.

KeEN, H., JARretT, R.J. \& Fuller, J.H. (1974) Tolbutamide and arterial disease in borderline diabetics. In: Diabetes. Proceedings of the Eighth Congress of the International Diabetes Federation, p. 588. Excerpta Medica, Amsterdam.

KeEN, H., TraCk, N.S. \& Sowry, G.S.C. (1975) Arterial pressure in clinically apparent diabetics. Diabète et Métabolisme, 1, 159.

Keen, H., Jarrett, R.J., Fuller, J.H., Ward, J.D. \& ZIMMET, P.H. (1975) Controlled trial of phenformin in borderline diabetics: five year findings. Presented to the European Association for the Study of Diabetes, Munich 1975. Diabetologia, 11, 355. Abstr. 141.

MARKS, H.H. (1971) Onset, course, prognosis and mortality in diabetes mellitus. In: Joslin's Diabetes Mellitus (11th Edition) (Ed. by A. Marble, P. White, R. F. Bradley \& L. P. Krall), p. 226. Lea \& Febiger, Philadelphia.

Mattock, M., Fuller, J.H. \& WHO Diabetes Group (1976) Plasma cholesterol esterifying enzyme in diabetics and normal controls. In: Aortic Disease C. Diabetes Mellitus (chap. 5), presented 10 May, European Society for Clinical Investigation, Rotterdam. (Submitted for presentation.)

Mitchell, J.R.A. \& Schwartz, C. (1965) Arterial Disease (1st Edition), p. 97 et seq. Blackwell, Oxford.

Morrison, A.D., Clements, R.S., JR \& Winegrad, A.I. (1970) Demonstration of the polyol pathway in the aortic wall. In: Atherosclerosis: Proceedings of the Second International Symposium (Ed. by R. J. Jones), p. 119. Springer-Verlag, Berlin.
O'Malley. B.C., Ward, J.D., Timberley, W.R., Porter N.R. \& Preston, F.E. (1975) Platelet abnormalities in diabetic peripheral neuropathy. Lancet, ii, 1274.

OsLer, W. (1912) The Principles and Practice of Medicine (8th Edition), p. 426. Appleton's New York \& London.

Pell, S. \& D'Alonzo, C.A. (1970) Factors associated with long-term survival of diabetics. Journal of the American Medical Association, 214, 1833.

PyKe, D.A. (1968) Arterial disease and diabetes. In: Clinical Diabetes and its Biochemical Basis (1st Edition) (Ed. by W. G. Oakley, D. A. Pyke \& K. R. Taylor), p. 503. Blackwell, Oxford.

Reaven, G.M., Lerner, R.L., Stern, M.P. \& Farquhar, J.W. (1967) Role of insulin in endogenous hyperglyceridemia. Journal of Clinical Investigation, 46, 1756.

Robertson, W.B. \& Strong, J.P. (1968) Atherosclerosis in persons with hypertension and diabetes mellitus. In: Geographical Pathology of Atherosclerosis (1st Edition) (Ed. by H. C. McGill, Jr). Williams and Wilkins, Baltimore.

Ross, R. \& Glomset, J.A. (1973) Atherosclerosis and the smooth muscle cell. Science, 180, 1332.

Schonfeld, G., Birge, C., Miller, J.P., Kessler, G. \& SANTiago, J. (1974) Apolipoprotein B levels and altered lipoprotein composition in diabetes. Diabetes, 23, 827.

Soler, N.G., Bennetr, M.A., Pentecost, B.L., Fitzgerald, M.G. \& Malins, J.M. (1975) Myocardial infarction in diabetics. Quarterly Journal of Medicine, 44, 125.

Stout, R.W. \& Vallance-OWEN, J. (1969) Insulin and atheroma. Lancet, i, 1078.

Stout, R.W. (1971) The effect of insulin on the incorporation of $\left(1-{ }^{14} \mathrm{C}\right)$ sodium acetate into the lipids of the rat aorta. Diabetologia, 7, 367.

Stout, R.W., Bierman, E.L. \& Ross, R. (1975) The effect of insulin on the proliferation of cultured primate arterial smooth muscle cells. Circulation Research, 36, 319.

Tzagournis, M., Chiles, R., Ryan, J.M. \& Skillman, T.G. (1968) Interrelationships of hyperinsulinism and hypertriglyceridemia in young patients with coronary heart disease. Circulation, 38, 1156.

University Group Diabetes Program (1970) A study of the effects of hypoglycemic agents on vascular complications in patients with adult-onset diabetes. I. Design, methods and baseline results. II. Mortality results. Diabetes, 19 (suppl. 2), 747.

University Group Diabetes Program (1975) A study of the effects of hypoglycemic agents on vascular complications in patients with adult-onset diabetes. V. Evaluation of phenformin therapy. Diabeles, 24 (suppl. 1), 65. 